\title{
Corollaries of Point Spread Function with Asymmetric Apodization
}

\author{
Naresh Kumar Reddy Andra, ${ }^{1,2}$ Udaya Laxmi Sriperumbudur, ${ }^{3}$ and Karuna Sagar Dasari ${ }^{2}$ \\ ${ }^{1}$ Department of Physics, CMR Institute of Technology, Medchal Road, Kandlakoya, Hyderabad, Telangana 501401, India \\ ${ }^{2}$ Optics Research Group, Department of Physics, University College of Science, Osmania University, No. 49, \\ Hyderabad, Telangana 500007, India \\ ${ }^{3}$ Department of Physics, Keshav Memorial Institute of Technology, Narayanguda, Hyderabad, Telangana 500029, India
}

Correspondence should be addressed to Naresh Kumar Reddy Andra; naarereddy@gmail.com

Received 5 August 2015; Revised 2 November 2015; Accepted 17 November 2015

Academic Editor: Ivan Moreno

Copyright (C) 2016 Naresh Kumar Reddy Andra et al. This is an open access article distributed under the Creative Commons Attribution License, which permits unrestricted use, distribution, and reproduction in any medium, provided the original work is properly cited.

Primary energy based corollaries of point spread function with asymmetric apodization using complex pupil function have been studied in the case of three-zone aperture. Merit function like semicircled energy factor, excluded semicircled energy, and displaced semicircled energy were analyzed with respect to Airy case in terms of phase and amplitude apodization. Analytical results have been presented for the optimum parameters of phase and amplitude asymmetric apodization.

\section{Introduction}

The principal corollaries measure the fraction of the total energy enclosed in the PSF of specific radius " $\delta$ " in the focal plane of optical imaging systems. Flux in a circle of specific radius (encircled energy) is the significant parameter in quantifying the performance of optical without aberrations. A lot of work has been done on the energy corollaries, but all the studies were pertained to symmetrically apodized PSF where the energy distribution on both sides of the diffraction centre is symmetric, whereas current study is dealing with asymmetric PSF. Asymmetric apodization is defined as the modification in the diffraction pattern by suppressing the optical side lobes on one side of the PSF at the cost of enhancing the side lobes on the other side while decreasing the width of the central maximum. For the obtained asymmetric PSF, where the central disc is not an exact circle, the corollaries based on the energy of the PSF, namely, the semicircled energy, excluded semicircled energy, displaced semicircled energy, and mean apodization ratio on both sides of the PSF, are investigated for the amplitude and phase filters.

Initially the idea of asymmetric apodization was introduced by Cheng and Siu $[1,2]$. Later Siu et al. continued their work and successfully applied asymmetric apodization to one-dimensional arrays [3]. All these studies are significant breakthrough in asymmetric apodization studies. Based on their studies Reddy and Sagar [4] applied the concept of asymmetric apodization to semicircular arrays of circular pupil functions and they achieved improved side-lobe suppression. Reddy and Sagar [5] introduced semicircled energy factor for analyzing the flux distribution in the image plane of optical systems with phase-only pupil. Naresh Kumar Reddy et al. [6] studied Strehl ratio, total transmission factor, and half power diameter for point spread function of imaging systems with asymmetric apodization. Rayleigh [7] discussed importance of the encircled factor to determine the illuminations in the edge ringing effect. Luneberg [8] studied third apodization problem to find the optimum pupil function for maximum encircled energy. Ueno and Asakura [9] investigated optimum pupil apodization for maximum encircled energy with specified overall transmittance. Mondal $[10,11]$ studied corollaries of PSF apodized with various amplitude filters. A few earlier studies in this case are limited [12-15]. Earlier studies [1-6] are the basis for current investigation. The present study on corollaries of the PSF of an asymmetric optical imaging system enables us to know the effects of asymmetric apodization on the consequences like increase in resolution in the Sparrow criterion, decrease in full width 
half maximum (FWHM), and so forth. Finally a comparison study can be made between Airy PSF corollaries and APSF corollaries from which important conclusions are presented.

\section{Theory}

Based on the Fresnel-Kirchhoff diffraction theory, amplitude impulse response of phase and amplitude apodized optical imaging systems can be given as

$$
A_{F}(0, u)=2 \int_{0}^{1} f(r) J_{0}(u r) r d r
$$

where $J_{0}(u r)$ is the Bessel function of the first kind and zero order with argument $(u r) . f(r)$ is the pupil function, where $u=(2 \pi / \lambda) \sin \theta$. Here $r$ is the radial coordinate in the complex pupil function. $f(r)$ for the three-zone amplitude and phase filter is given by

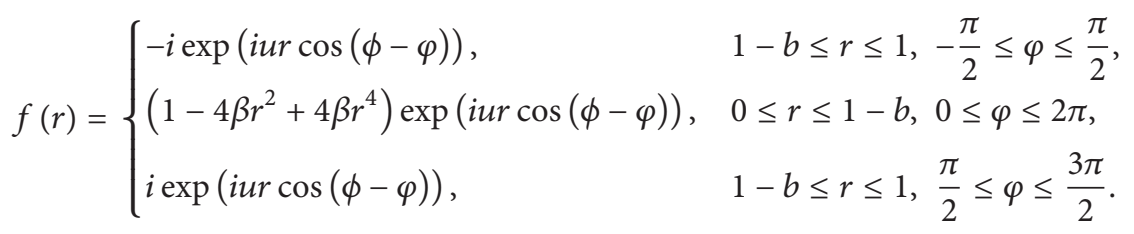

On introducing the amplitude apodization parameter $\beta$ to control the degree of nonuniformity of transmission in the central region of the pupil of radius $(1-b)$ and introducing semicircular edge ring of width $b$ to control the phase apodization of the pupil function, complex pupil function $f(r)$ consists of three zones with two semicircular edge rings of equal width " $b$ " with opposite phase transmittances of the form $+i$ and $-i$ and the central circular zone is with amplitude apodizer and its corresponding phase transmittance is zero. For amplitude apodizer, the amplitude transmittance at the center $(r=0)$ of aperture $f(r)$ is equal to unity, that is, maximum. It decreases towards the edge of the aperture as " $r$ " goes to 0.5 for all values of $\beta$. From Figure 2, it is clear that $f(r)$ is minimum at edges for any degree of amplitude apodization. $\beta$ is the apodization parameter controlling the degree of nonuniformity of the transmittance over zone of radius $(1-b)$. The range it takes is $0 \leq \beta \leq 1$. It is clear that, for $\beta=0$, the transmittance of this zone is uniform. Transmittance over the rest of the two zones of circular aperture of unit radius is unity. Here $b$ is the certain width of semicircular edge ring. The range of values it takes is $0 \leq b \leq$ 0.1 . It is obvious that, for $b=0$, the PSF obtained is symmetric in nature. The asymmetry in the PSF increases with the values of $b$ (degree of phase apodization) and further improved by increase in amplitude apodization parameter $\beta$. The design of pupil function can be seen in Figure 4.

The optics term semicircled energy refers to a measure of concentration of energy over one side of the PSF from the diffraction centre. Calculation of the semicircled energy factor (SCEF) on both sides of the PSF gives the total distribution of energy in the asymmetric PSF. In the case of two-dimensional aperture, the side-lobe region is specified not only by " $u$," which related to the orientation $\theta$, but also by azimuth angle $\Phi$, which determines how large the good side "window" is, taken as $-\pi / 3<\Phi<\pi / 3$. By the minimum of intensity square, the semicircular edge ring width is determined to be $b=0.1 . A_{F}(0, u)$ is the amplitude in the image plane at point " $u$ " (reduced dimension less diffraction coordinate), units away from the diffraction head due to the pupil function. Hence, the integration over $\Phi$ introduces just the same constant in both the numerator and the denominator. Amplitude contains Bessel functions of the first kind, which oscillate from positive to negative values very rapidly and become zero at a finite distance from the centre of the diffraction image $(u=0)$. Thus

$$
\begin{aligned}
\operatorname{SCEF}(\delta)_{\text {Good Side }}= & \frac{\int_{0}^{\delta}\left[\int_{0}^{1-b} f(r) J_{0}(u r) r d r\right] u d u-i \int_{1-b}^{1} \int_{-\pi / 2}^{\pi / 2} \exp (i u r \cos (\phi-\varphi)) r d r d \varphi}{\int_{0}^{1}|f(r)|^{2} r d r} \\
\operatorname{SCEF}(\delta)_{\text {Bad Side }}= & \frac{\int_{0}^{-\delta}\left[\int_{0}^{1-b} f(r) J_{0}(u r) r d r\right] u d u+i \int_{1-b}^{1} \int_{\pi / 2}^{3 \pi / 2} \exp (\operatorname{iur} \cos (\phi-\varphi)) r d r d \varphi}{\int_{0}^{1}|f(r)|^{2} r d r}
\end{aligned}
$$

Excluded semicircled energy (ESCE) is complementary quantity of the semicircled energy. In order to examine the outer ring structure in detail, this factor is convenient as compared to the semicircled energy. The function of an 
asymmetric apodizer is best fulfilled if this factor is maximum on good side and minimum on bad side of the obtained asymmetric PSF.

The expression for excluded semicircled energy is as follows:

$$
\begin{aligned}
& \operatorname{ESCE}(\delta)=1-\operatorname{SCEF}(\delta), \\
& \operatorname{ESCE}(\delta)=\frac{\int_{R}^{ \pm \delta}\left|A_{F}(0, u)\right|^{2} u d u}{\int_{0}^{\infty}\left|A_{F}(0, u)\right|^{2} u d u} .
\end{aligned}
$$

This energy corollary is useful in some photometric situations. For example, the contrast at the center of the image of a black disk seen against a uniform incoherent background is semicircled energy, so excluded semicircled energy gives the residual intensity. Such situation exists in the transit of planet across the Sun. this parameter is useful in evaluating apodization for suppressing the ring structure. Displaced semicircled energy (DSCE) is the difference between the semicircled energy (SCEF) of the perfect system (Airy) and given case (asymmetrically apodized). It is useful to compare the energy distribution in the actual optical system to its perfect counterpart. Thus

$$
\operatorname{DSCE}(\delta)=\operatorname{SCEF}_{A}(\delta)-\operatorname{SCEF}_{F}(\delta),
$$

where $\operatorname{SCEF}_{A}(\delta)$ gives the semicircled energy for Airy case and $\operatorname{SCEF}_{F}(\delta)$ gives the semicircled energy for asymmetrically apodized case. If the displacement semicircled energy is positive, there is an outward displacement of energy, and if it is negative, there is an inward displacement of energy. This parameter is more sensitive to aperture obscuration and less sensitive to image motion.

\section{Results and Discussions}

The improved resolution of asymmetric point spread function with amplitude and phase filters has been analyzed in terms of energy corollaries on both sides of the main lobe. However, we reported the majority of the results for the good side which is the half part of the complete pattern. The results on investigations on semicircled, excluded, and displaced energies have been studied. In order to investigate the flux enclosed in the detector plane of optical imaging, systems have been obtained as function of dimension less diffraction parameter " $u$ " by employing the standard numerical method of integration. An iterative method has been developed and applied to determine the asymmetric radial distribution of energy within a specific radius " $\delta$ " in the plane of observation or detection.

Semicircled energy factor of the APSF as function of " $\delta$ " for various combinations of $b$ and $\beta$ is obtained for Airy case $(b=0$ and $\beta=0)$, symmetric apodized case $(b=0$ and $\beta \neq$ $0)$, and asymmetric apodized case $(b \neq 0)$. In our analysis, it has been observed that, for Airy case, $\operatorname{SCEF}(\delta)$ increases rapidly for first few values " $\delta$ " in the receiver plane and later on slows down considerably before finally approaching half of the unity asymptotically.

In this case, there is quick increase in SCEF up to a value of $\delta$ around 3.0 which results in a fast growth in the peak

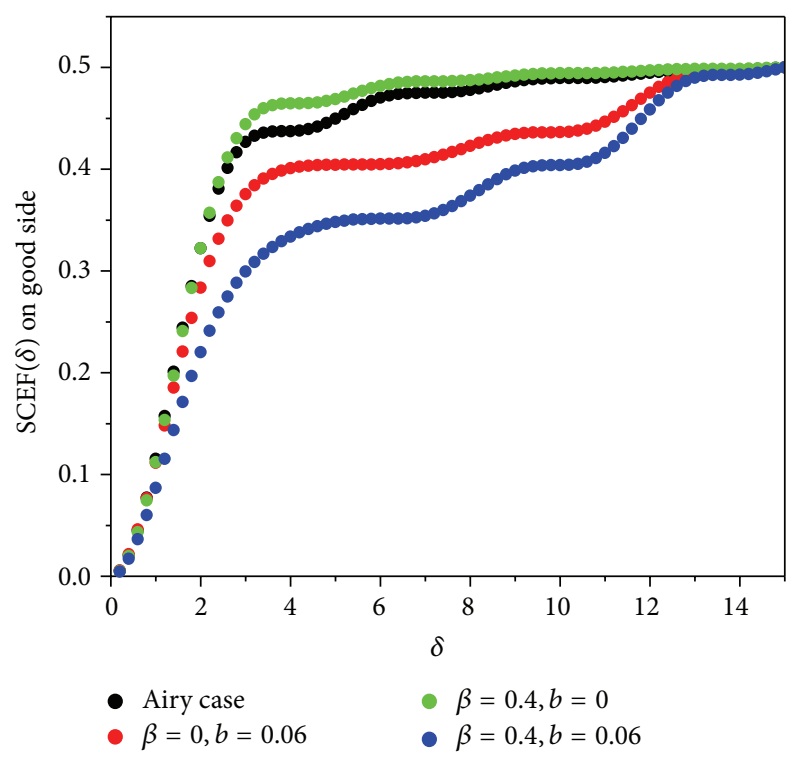

FIGURE 1: Semicircled energy on good side for different values of $b$ and $\beta$.

intensity of the main lobe. For $\beta=0.4$ and $b=0$, it has been observed that with the introduction of three-zone amplitude filter in the central region of the aperture there is a variation in SCEF compared to Airy case. Till $\delta$ is equal to 3.75 the semicircled energy is equal to that of Airy case and beyond 3.75 the semicircled energy is greater than the Airy case. Two equal semicircular edge rings of width $b(0.06)$ with opposite transparencies have been distributed over the central circular region of the aperture with which an asymmetric PSF has been obtained with unequal energies on both sides of the central lobe. The side on which the side lobes are enhanced is referred to as bad side and the other is referred to as good side. From Figure 1, it is clear that for all the values of $\delta$ varying from 0 to 15 the semicircled energy is found to be less than other cases (Airy case and also symmetrically apodized case).

From Figure 1, it has been observed that the value of SCEF is not as much of Airy case. It is also noticed that with the introduction of amplitude apodizer in the central region of the aperture the semicircled energy factor is further decreased comparatively with the above-mentioned cases. Figure 2 shows the variation in amplitude transmittance for different values of amplitude apodization parameter $\beta$ as a function of the radial coordinate " $r$ " of the pupil. It shows a monotonic decrease of amplitude transmittance $t(r)$ with the normalized distance $r$. At the center of the pupil $(r=0)$ transmittance is unity, that is, highest for all values of $\beta$, and it decreases towards the edges as $r$ goes to 0.5 . Transmittance is minimum at the edges for all values of $\beta$. Figure 3 explains the study of SCEF on both sides of the PSF for $b=0$, while $\beta$ varies from 0 to 1 . In this case the semicircled energy on both sides of the central lobe is symmetric or uniform. Hence the results obtained on the bad side are similar to those of the good side. It has been observed that the lowest values of semicircled energy are obtained for $\beta=0$ and the highest values have been obtained for $\beta=1$. It is clear that 


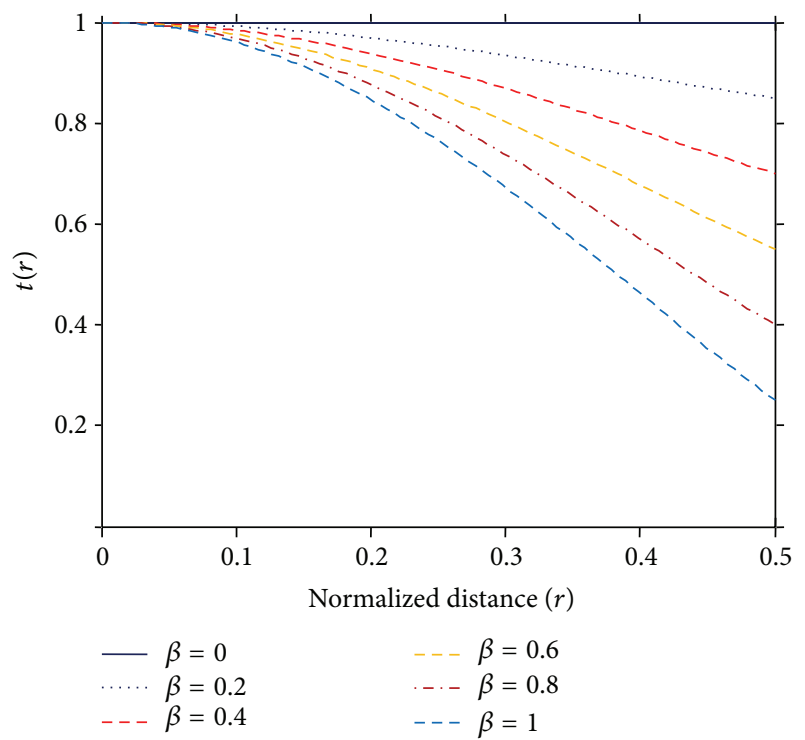

FIGURE 2: Amplitude transmittance of the amplitude apodizer decreases with radial coordinate.

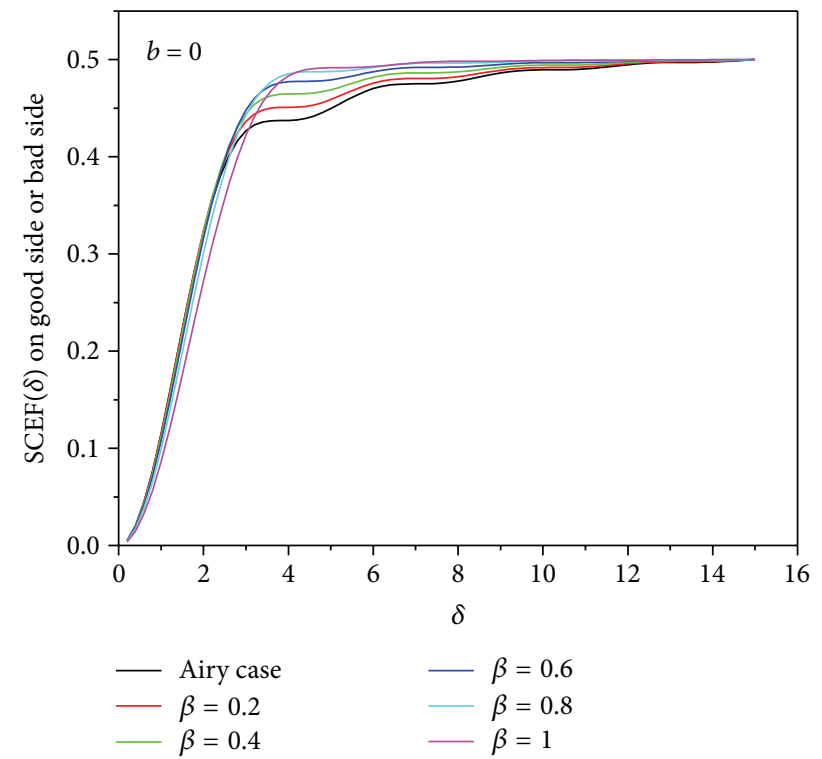

Figure 3: Semicircled energy on good side or bad side for various values of $\beta$ when $b=0$.

the semicircled energy vanishes at the origin $(\delta=0)$ and increases monotonically, approaching half of the unity. The semicircled energy on good side is decreasing with increasing the value of $b$. For all values of $\delta$ varying from 0 to 15 it is observed that the semicircled energy value is less than Airy case, whereas on bad side semicircled energy obtained its maximum factor which is more than Airy case for $b=0.02$ and 0.04 . On further rise in semicircular edge ring width above $0.04 \operatorname{SCEF}(\delta)$ on bad side decreases.

The values of the excluded semicircled energy for various values of $\delta$ as function of $b$ and $\beta$ are obtained. It has computed quite easily from SCEF. For $b \neq 0(0.06)$ and $\beta=0$

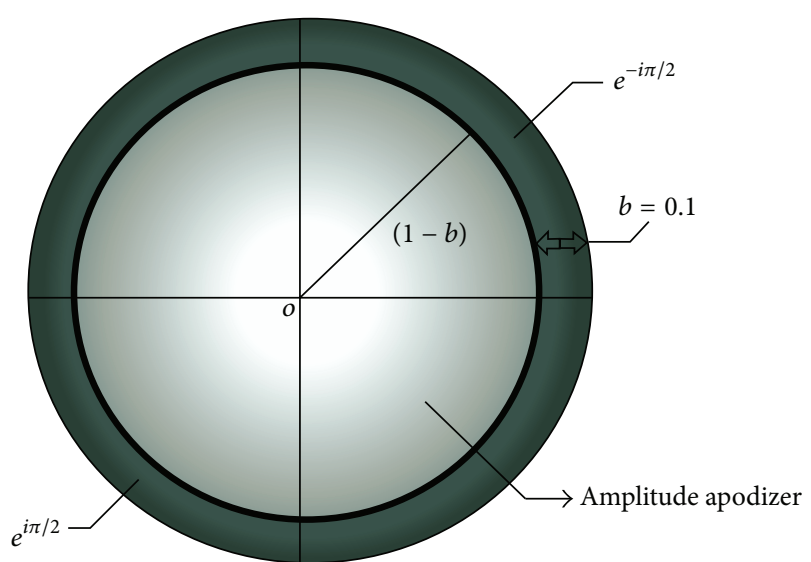

FIGURE 4: Generalized scheme of complex pupil function.

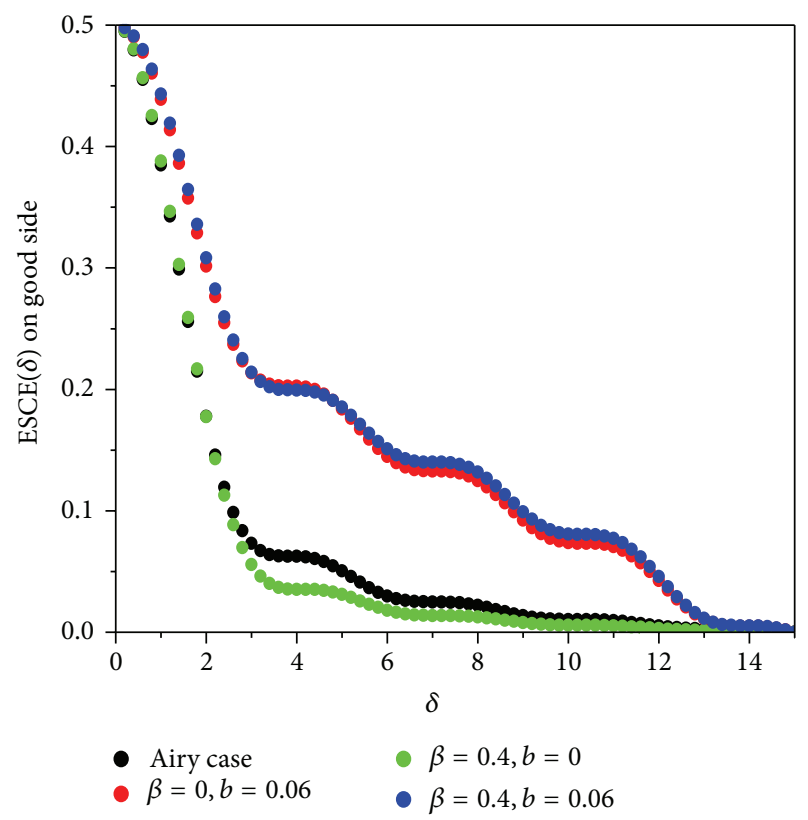

FIGURE 5: Excluded energy on good side for different values of $b$ and $\beta$.

or $\beta \neq 0(0.4)$, more excluded energy has been recorded than that of Airy case, whereas for symmetrically apodized case $(\beta \neq 0$ and $b=0)$ the excluded energy is detected less than Airy case. It has been clearly illustrated in Figure 5. It is concluded that with increase in $\beta$ value the excluded energy is decreasing. It is depicted clearly in Figure 6. With increase of degree of amplitude apodization in the central region of the aperture, the central disc widens; more energy is accumulated in the central region compared to the energy concentrated in the near vicinity of the diffraction centre, whereas in the case of asymmetric apodization the energy is shifting to one side of the diffraction centre (bad side) and simultaneously narrowing the central lobe of the diffraction pattern. The side on which the optical lobes are suppressed is termed as good side and the energy from this side has been excluded more to the outer rings. 


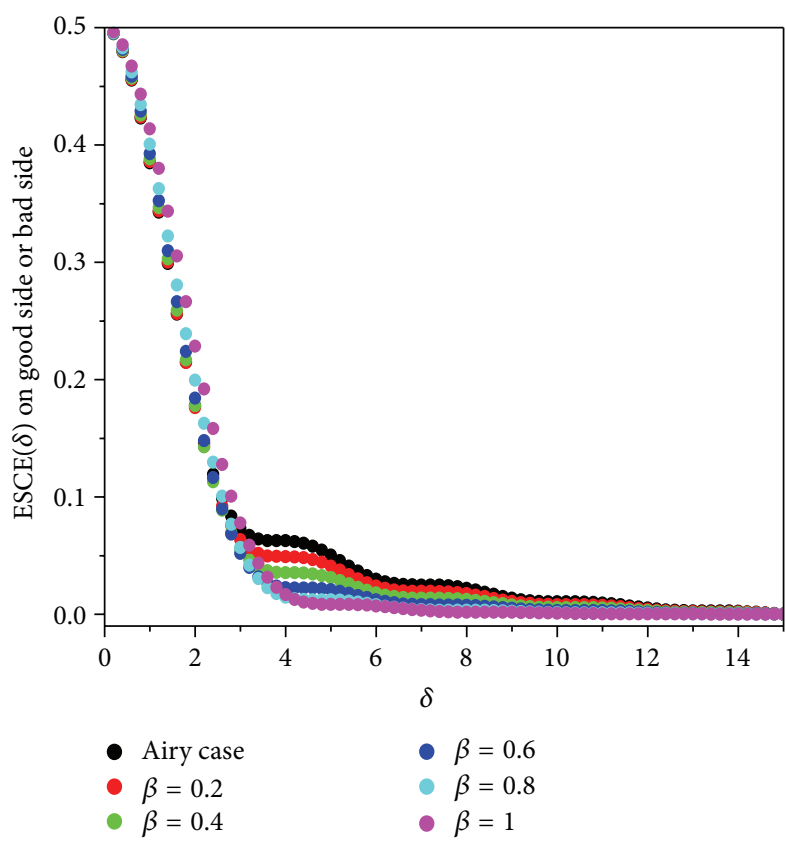

FIgURE 6: Excluded energy on good side or bad side for various values of $\beta$ when $b=0$.

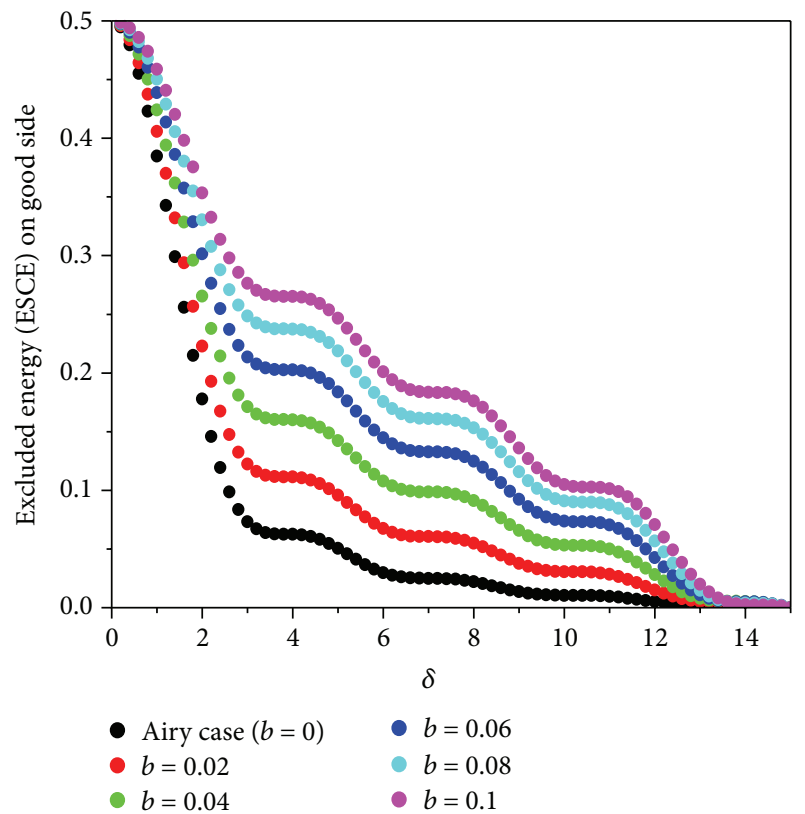

FIGURE 7: Excluded energy on good side for various amount of asymmetric apodization $(b)$ when $\beta=0$.

The excluded energy on the good side increases with increase in the value of " $b$." It is observed that it is minimum for $b=0$ and maximum for $b=0.1$. It is observed in more detail in Figure 7.

The values of displaced semicircled energy on good side for various combinations of $b$ and $\beta$ are studied. There is no displaced energy for Airy case by definition. It has been observed that for symmetrically apodized optical system

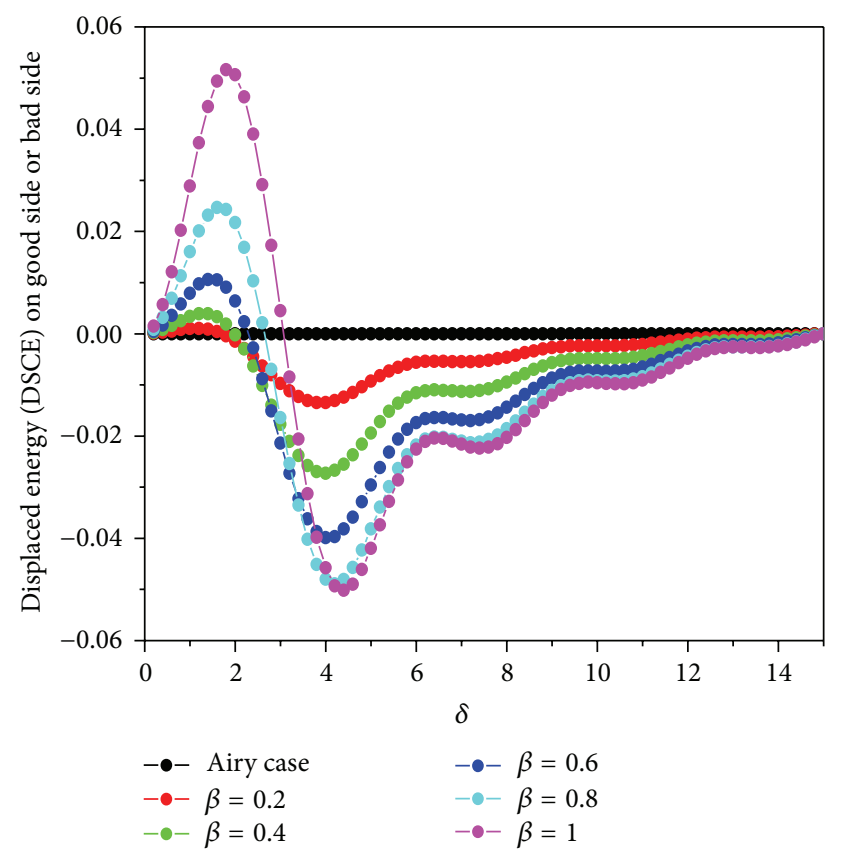

FIGURE 8: Displaced energy on good side or bad side for various values of $\beta$ when $b=0$.

$(\beta \neq 0$ and $b=0)$ the displaced energy is negative, which means that the semicircled energy possesses higher values. Hence the displaced energy is lying on the negative axis. For an optical system $(\beta=0$ and $b \neq 0)$ the semicircled energy on the good side is less than Airy case; hence by definition the values of displaced energy are positive for all the values of $\delta$. For $\beta \neq 0$ and $b \neq 0$, the semicircled energy of the system is very low compared to the semicircled energy in Airy case; hence the displaced energy values are high compared to the other cases. Figure 8 illustrates that in absence of phase apodization $(b=0)$ for all values of $\beta$ the displaced energy is lower than Airy case for lower values of $\delta$ and on further rise in $\delta$ the displaced energy is on negative axis. Negative values of displaced energy in Figure 8 indicate that the semicircled energy increases with degree of amplitude apodization $(\beta)$. Figure 9 shows the corresponding distribution of diffraction filed, where the Airy PSF is presented as a solid black curve. It is clearly seen that the complex pupil under different considerations obtains the optical side lobes much lower than Airy ones and the central lobe is narrowed.

\section{Conclusions}

The following explanation for the energy based corollaries of asymmetric point spread function can be given. With introduction of amplitude apodizer in the central region gives modified PSF with equal energies (equal intensity in side lobes or secondary maxima) on both sides of the diffraction centre. Hence, as $\beta$ increases, the semicircled energy on both sides of the PSF increases. In the case of asymmetric 


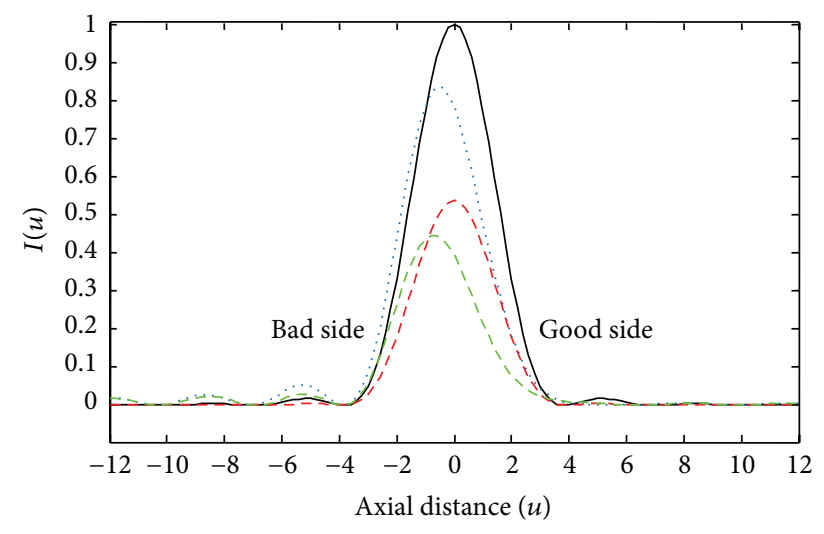

$\ldots .$. Asymmetric $(b=0.06$, phase only)
--- Asymmetrically apodized (phase and amplitude)
_ Airy case (or) unapodized
--- Symmetrically apodized $(\beta=0.4$, amplitude only)

FIgURE 9: The point spread function of a complex pupil filter under different conditions.

apodization, as $b$ increases, the energy distribution on both sides of the diffraction centre is not equal (i.e., asymmetric). With increase in $b$ value the semicircled energy decreases on the good side and at the same time for $b=0.02$ and 0.04 the semicircled energy on bad side is more than that of Airy case. Hence the excluded energy increases on good side of the APSF as degree of asymmetric apodization increases. Increase in the semicircled excluded energy results in the enhancement of resolution of the optical system. On the contrary, as $\beta$ increases, the amount of excluded energy decreases. Hence the highest excluded energy is recorded for maximum amplitude apodization $(\beta=1)$. For lower values of $\delta$, the positive values of displaced energy are obtained which indicates more energy in the Airy pupil than that of the apodized pupil with complex pupil filters. The displaced energy on good side of the diffraction centre increases as $b$ increases rendering the asymmetrically apodized pupils energy lower than Airy pupils. In this case, the energy is shifting to one side of the diffraction centre (i.e., bad side of the PSF) and simultaneously narrowing the central lobe of the diffraction pattern. The side on which the optical lobes are suppressed is termed as good side and the energy from this side has been excluded more to the outer rings. This asymmetry increases with the width $(b)$ of the edge rings and is promoted by the increase in the degree of apodization $(\beta)$ of the central circular region of the aperture. Thus, present study has many applications in diverse potential fields such as confocal microscopy, spectroscopy, astronomy, communication, and medical imaging.

\section{Conflict of Interests}

The authors declare that there is no conflict of interests regarding the publication of this paper.

\section{Acknowledgment}

The authors are thankful to Anup L. Shah, Scientist-H (Project Director, CHESS, RCI campus, DRDO), Hyderabad, for technical support and some interesting suggestions concerning the bibliographic background.

\section{References}

[1] L. Cheng and G. G. Siu, "Asymmetric apodization," Measurement Science and Technology, vol. 2, no. 3, pp. 198-202, 1991.

[2] G. G. Siu, L. Cheng, D. S. Chiu, and K. S. Chan, "Improved sidelobe suppression in asymmetric apodization," Journal of Physics D: Applied Physics, vol. 27, no. 3, pp. 459-463, 1994.

[3] G. G. Siu, M. Cheng, and L. Cheng, "Asymmetric apodization applied to linear arrays," Journal of Physics D: Applied Physics, vol. 30, no. 5, pp. 787-792, 1997.

[4] A. N. K. Reddy and D. K. Sagar, "Point spread function of optical systems apodized by semi circular arrays of $2 \mathrm{D}$ aperture with asymmetric apodization," Journal of Information and Communication Convergence Engineering, vol. 12, no. 2, pp. 8388, 2014.

[5] A. N. K. Reddy and D. K. Sagar, "Semi circled energy of asymmetrically apodized optical systems," Advances in Applied Science Research, vol. 5, no. 2, pp. 42-48, 2014.

[6] A. Naresh Kumar Reddy, R. Komala, M. Keshavulu Goud, and S. Lacha Goud, "A few PSF-based corollaries of optical systems apodised asymmetrically with two-dimensional complex pupil filters," Armanian Journal of Physics, vol. 4, no. 4, pp. 200-205, 2011.

[7] L. Rayleigh, "Investigations in optics, with special reference to the spectroscope," Philosophical Magazine Series 5, vol. 8, no. 49, pp. 261-274, 1879.

[8] P. Luneberg, "Measurement of contrast transmission characteristics in optical image formation," Optica Acta, vol. 1, no. 2, pp. 80-89, 1954.

[9] T. Ueno and T. Asakura, "Apodization for maximum encircled energy with specified over-all transmittance," Journal of Optics, vol. 8, no. 1, pp. 15-31, 1977.

[10] K. P. Rao, P. K. Mondal, and T. Seshagiri Rao, "Diffracted field characteristics of Straubel class of apodisation filters," Pramana, vol. 7, no. 6, pp. 389-396, 1976.

[11] K. Surendar, S. L. Goud, and P. K. Mondal, "PSF corollaries of apodized optical systems," Acta Ciencia Indica, vol. 18, no. 1, pp. 91-96, 1992.

[12] E. C. Kintner, "Calculating the encircled energy in the pointspread function," Optica Acta: International Journal of Optics, vol. 24, no. 10, pp. 1075-1076, 1977.

[13] J. Campos, F. Calvo, and M. J. Yzuel, "Resolving power and encircled energy in aberrated optical systems with filters optimized for the Strehl ratio," Journal of Optics, vol. 19, no. 3, pp. 135-144, 1988.

[14] A. A. Dantzler, "Encircled energy correction method for raytrace programs," Applied Optics, vol. 27, no. 24, pp. 5001-5002, 1988.

[15] S. J. Park and C. S. Chung, "Influence of Bessel and Bessel-Gauss beams on the point spread function and the encircled energy," Journal of the Korean Physical Society, vol. 30, no. 2, pp. 194-201, 1997. 

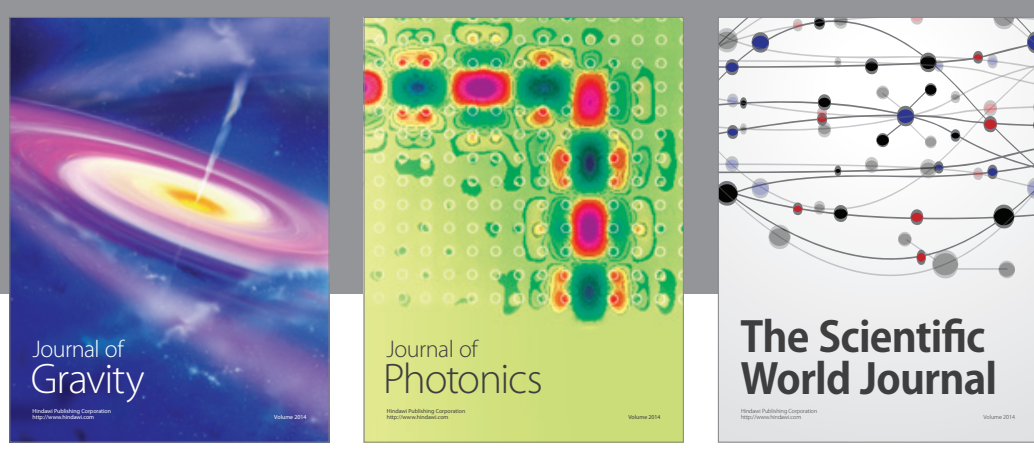

The Scientific World Journal
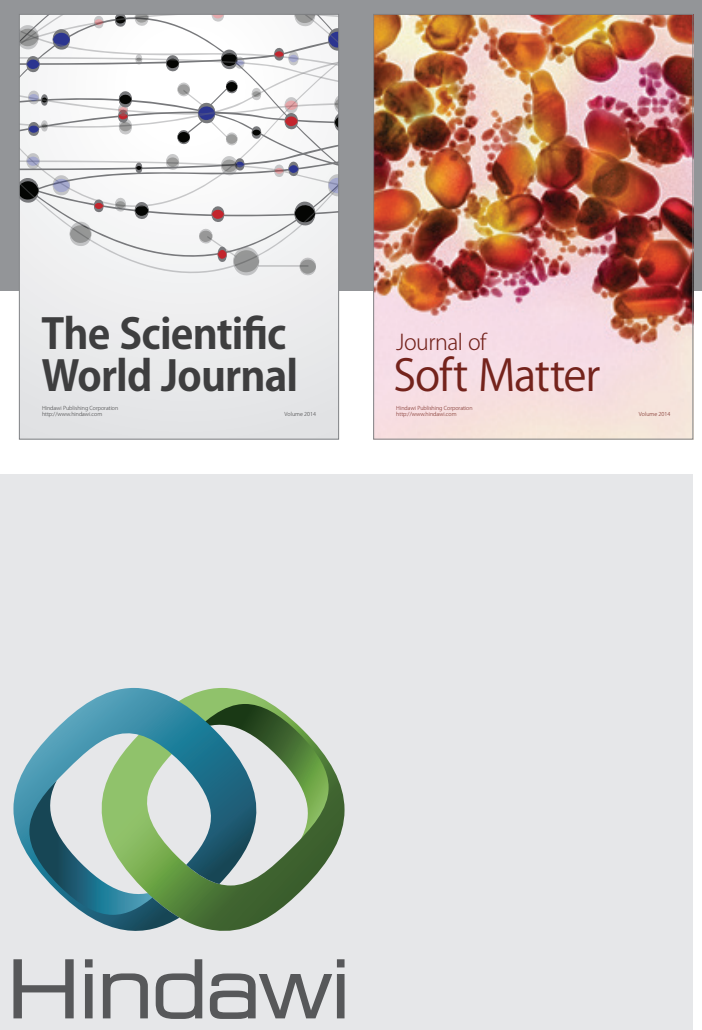

Submit your manuscripts at

http://www.hindawi.com

nternational Journal of

Statistical Mechanics
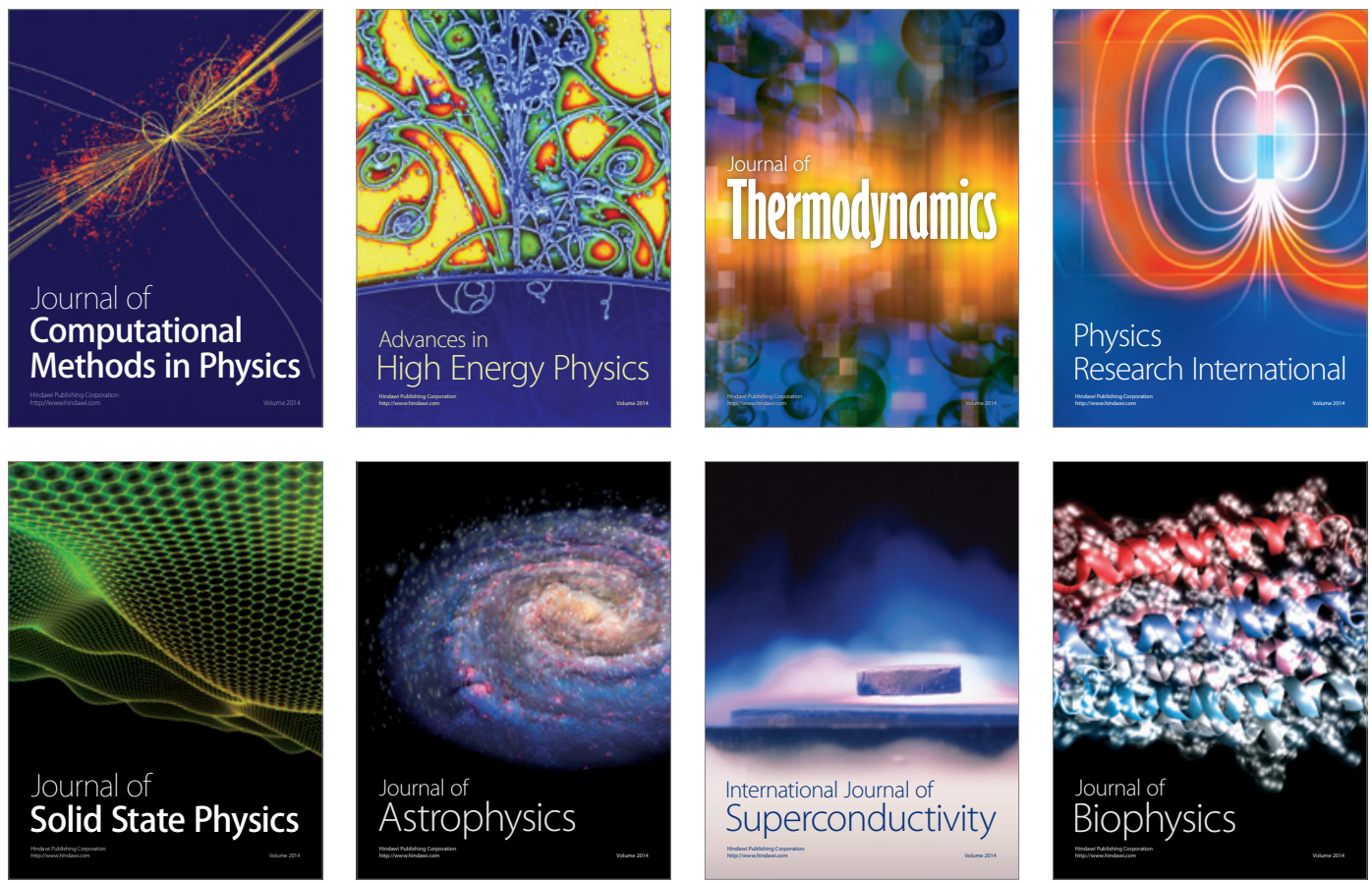
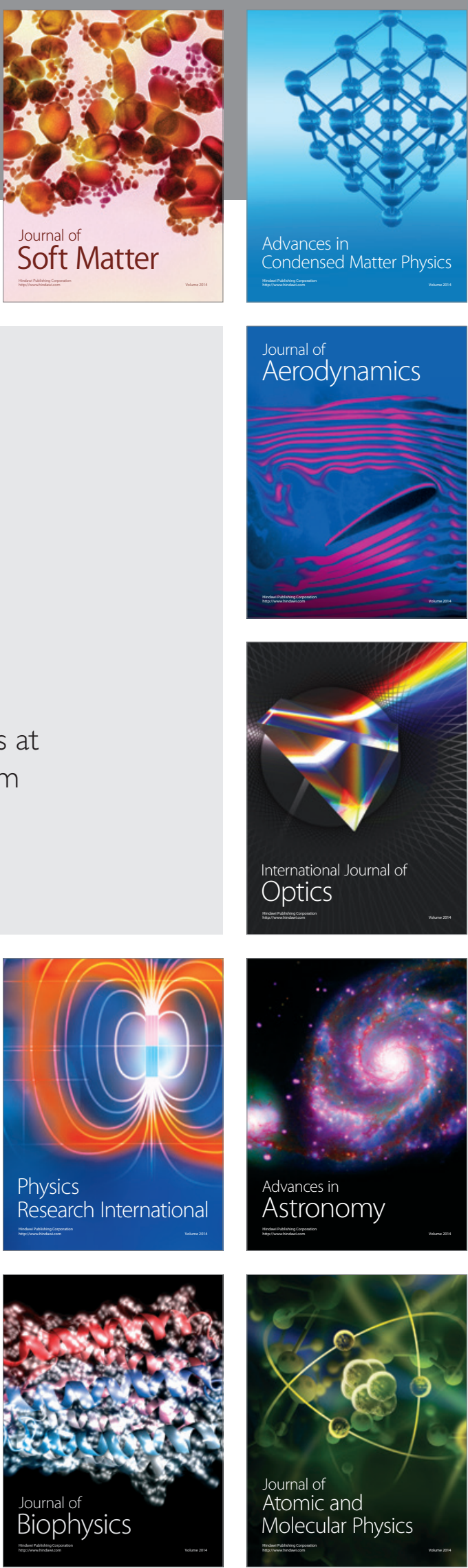